International Journal of Pure and Applied Mathematics

Volume 94 No. 4 2014, 589-599

ISSN: 1311-8080 (printed version); ISSN: 1314-3395 (on-line version)

url: http://www.ijpam.eu

doi: http://dx.doi.org/10.12732/ijpam.v94i4.15

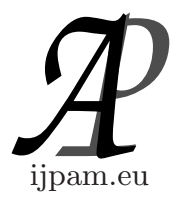

\title{
MATHEMATICAL MODELING OF ELECTROMAGNETIC RESPONSE FOR CONDUCTIVE CIRCULAR CYLINDER BODY EMBEDDED IN GROUND WITH NORMAL CURVE CONDUCTIVITY PROFILE
}

\author{
P. Haarsa \\ Department of Mathematics \\ Srinakharinwirot University \\ Bangkok, 10110, THAILAND
}

\begin{abstract}
The purpose of this paper is to give a mathematical model and methods for uncovering the conductivity, the conductance, the thickness, and the depth of a circular body buried under the ground surface with a normal curve conductivity profile. The solution of electric field for this model earths structure is obtained by using the Hankel transforms and integral equations. The Cauchy-Euler method and the method of variation of parameter are introduced. Chave's algorithm is used for numerical computing the inverse Hankel transforms of the electric field solution. The operations on matrices are applied for resolving the linear system of equations. The conducts of electric field are shown in graphical forms.
\end{abstract}

AMS Subject Classification: $86 \mathrm{~A} 25$

Key Words: electromagnetic field, normal curve, Chave's algorithm, Hankel transforms

\section{Introduction}

The electromagnetic method is the most ordinarily employed in geophysical

Received: May 4, 2014

(C) 2014 Academic Publications, Ltd. url: www.acadpubl.eu 
exploration because it reacts best to good electrical conductors at the near surface to as deep as many kilometers under the earth surface, and also it is less expensive correlating to other exploration techniques. Lee and Ignetik [4] considered the forward problem of the transient electromagnetic response of a half-space with an exponentially varying conductivity profiles. They indicated out that the conductivity variation of the ground may sometimes be reasonably approximated by an exponential variation since soil salinity profiles frequently show monotonically increasing or decreasing salt conductivity of the ground. Ketchanwit [3] studied time domain electromagnetic response in heterogeneous media. Yooyuanyong and Siew [7] gave the mathematical model of electromagnetic response of a disk beneath an exponentially varying conductive overburden. Yooyuanyong and Chumchob [6] derived mathematical modelling of electromagnetic sounding for a conductive 3-D circular cylinder body embedded in a conducting half-space. The intention of this paper is to acquaint with a mathematical model and methods for studying the structure of the earth which has a circular cylinder body buried under the ground. We limit the conductivity to be denoted by $\sigma(z)=\sigma_{0} e^{-b(z-l)^{2}}$, where $\sigma_{0}$ a is positive constant, $b$ is constant, and $l$ is positive which is used to locate the peak of the bulge. The conductivity ground profile used in this paper is distinct from the models used by Lee and Ignetik [4] and Yooyuanyong and Siew [7], Ketchanwit [3] and Yooyuanyong and Chumchob [6].

\section{Derivation of Electromagnetic Field}

To formulate the problem, we regard a cylindrical polar coordinates $(r, \theta, z)$ which is introduced with $z>0$ and is taken vertically positive downwards. In addition, the origin under the center of the horizontal circular loop of height $h$ above the ground surface is used. The azimuthally symmetry yields merely electric field $E_{\theta}$, and magnetic fields $H_{r}$, and $H_{z}$ components. Following Morrison et al., [5] and Yooyuanyong [8], these field quantities are established to satisfy Maxwell's equations [2] in the form of equations

$$
\begin{gathered}
i \omega \mu H_{r}=-\frac{\partial E}{\partial z}, \\
i \omega \mu H_{z}=\frac{1}{r} \frac{\partial}{\partial r}(r E),
\end{gathered}
$$

and

$$
\frac{\partial H_{r}}{\partial z}-\frac{\partial H_{z}}{\partial r}=(\sigma(z)-i \omega \varepsilon) E+J_{s}
$$


where $i=\sqrt{-1}$ is an imaginary number, $J_{s}=a I(\omega) \delta(r-a) \delta(z+h) / r$ is the source current density, $\omega$ is the angular frequency, $\delta$ is the delta function, $\sigma(z)$ is the electrical conductivity of medium depending on depth only, $\varepsilon$ is the electric permittivity of medium, $\mu$ is the magnetic permeability of medium, and $I(\omega)$ is the current in a coil of a small radius $a$. Eliminating $H_{r}$ and $H_{z}$ from above equations lead to get the differential equation for electric fields as.

$$
i \omega \mu J_{s}=-\frac{\partial^{2} E}{\partial z^{2}}-\frac{\partial^{2} E}{\partial r^{2}}-\frac{1}{r} \frac{\partial E}{\partial r}+\frac{E}{r^{2}}-\left(i \omega \mu \sigma(z)+\omega^{2} \mu \varepsilon\right) E .
$$

By applying Hankel transforms which is defined as

$$
\widetilde{E}(\lambda, z, \omega)=\int_{0}^{\infty} r J_{1}(\lambda r) E(r, z, \omega) d r,
$$

where $J_{1}$ is Bessel function of the first kind of order 1, and equation (4) becomes

$$
-\frac{\partial^{2} \widetilde{E}}{\partial z^{2}}+\left[\lambda^{2}-\left(i \omega \mu \sigma(z)+\omega^{2} \mu \varepsilon\right)\right] \widetilde{E}=i \omega \mu a I(\omega) \delta(z+h) J_{1}(\lambda a) .
$$

We study the ground which has a circular body buried under the ground with a normal curve conductivity profile and given by $\sigma(z)=\sigma_{0} e^{-b(z-l)^{2}}$, where $\sigma_{0}$ a is positive constant, $b$ is constant, and $l$ is positive which is used to locate the peak of the bulge. We consider a primary alternating source current carried by a coil of radius $a$, at $z=-h$ above the surface of the ground $z=0$, (see Figure 1). At $z=z_{1}$, there is an object embedded in the ground.

\section{The Electric Field in Air}

The electric field in air can be denoted by $\widetilde{E}_{a i r}(\lambda, z, \omega)$ and expressed as the sum of two components

$$
\widetilde{E}_{a i r}(\lambda, z, \omega)=\widetilde{E}_{\text {air }}^{p}(\lambda, z, \omega)+\widetilde{E}_{\text {air }}^{s}(\lambda, z, \omega),
$$

where $\widetilde{E}_{\text {air }}^{p}(\lambda, z, \omega)$ is the primary electric field, and $\widetilde{E}_{\text {air }}^{s}(\lambda, z, \omega)$ is the secondary electric field. Both electric fields can be obtained from equation (5). In air, $\sigma_{\text {air }}(z) \cong 0$ and the electric field is given by [7]

$$
\widetilde{E}_{a i r}(\lambda, z, \omega)=\frac{i \omega \mu_{A} a I(\omega) J_{1}(\lambda a) e^{-\lambda|z+h|}}{2 \lambda}+v e^{\lambda z}, z \leq 0,
$$

where $\mu_{A}$ is the magnetic permeability of air and $v$ is an arbitrary constant. The electric field remains bounded as $z \rightarrow-\infty$, and $v$ is an arbitrary constant to be determined from the boundary condition. 


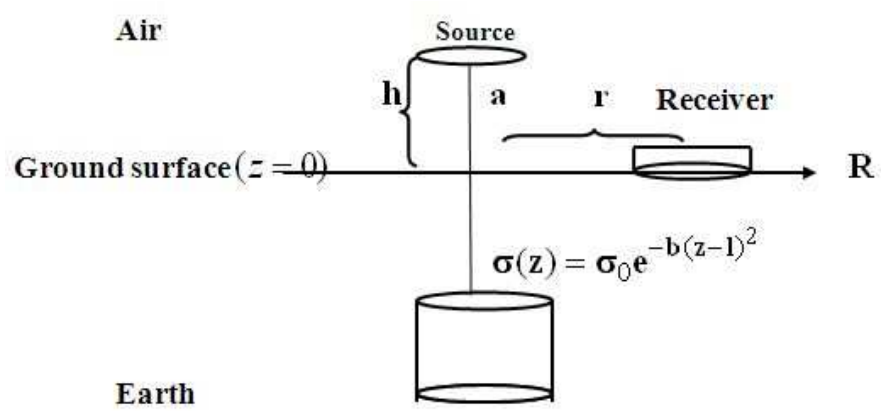

Figure 1: Geometric model of the problem.

\section{The Electric Field in Ground}

We denote that $E_{\text {ground }}$ is the total of electric field in ground which is denoted by

$$
E_{\text {ground }}=E_{g 1}+E_{g 2}
$$

where $E_{g 1}$ is the electric field response in ground without object and $E_{g 2}$ is the electric field response in ground with object. Using equation (4), we obtain the partial differential equation of $E_{g 1}$ and $E_{g 2}$, respectively, as follow

$$
\begin{aligned}
& \frac{\partial^{2}}{\partial z^{2}} E_{g 1}(r, z, \omega)+\frac{\partial^{2}}{\partial r^{2}} E_{g 1}(r, z, \omega) \\
& +\frac{1}{r} \frac{\partial}{\partial r} E_{g 1}(r, z, \omega)-\frac{1}{r^{2}} E_{g 1}(r, z, \omega)=k_{g}^{2} E_{g 1}(r, z, \omega),
\end{aligned}
$$

and

$$
\begin{aligned}
& \frac{\partial^{2}}{\partial z^{2}} E_{g 2}(r, z, \omega)+\frac{\partial^{2}}{\partial r^{2}} E_{g 2}(r, z, \omega)+\frac{1}{r} \frac{\partial}{\partial r} E_{g 2}(r, z, \omega) \\
& -\frac{1}{r^{2}} E_{g 2}(r, z, \omega)+k_{g}^{2} E_{g 2}(r, z, \omega)=i \omega \mu_{0} \sigma_{g 2} E_{o b j}
\end{aligned}
$$

where $k_{g}^{2}=i \omega \mu_{g} \sigma(z)+\omega^{2} \mu_{g} \varepsilon_{g}, E_{o b j}$ is the electric field on the object, $\mu_{g}$ is the magnetic permeability of ground, $\varepsilon_{g}$ is the electric permittivity of ground and $\sigma(z)=\sigma_{0} e^{-b(z-l)^{2}}$ is the conductivity of ground. Taking Hankel transforms to equations (8) and (9), we have

$$
\frac{d^{2}}{d z^{2}} \widetilde{E}_{g 1}(\lambda, z, \omega)-\lambda^{2} \widetilde{E}_{g 1}(\lambda, z, \omega)=0 .
$$


and

$$
\frac{d^{2}}{d z^{2}} \widetilde{E}_{g 2}(\lambda, z, \omega)-\lambda^{2} \widetilde{E}_{g 2}(\lambda, z, \omega)=-i \omega \mu_{g} \int_{0}^{\infty} s \sigma(z) E_{o b j}(s, z, \omega) J_{1}(\lambda s) d s
$$

Using the exponential substitution $\xi=e^{-b z / 2}$ to equation (10), we obtain the solution of equation (10) and given by

$$
\widetilde{E}_{g 1}(\lambda, \xi, \omega)=\eta_{1} \xi^{\phi}+\eta_{2} \xi^{-\phi}
$$

The Cauchy-Euler equation method and the method of variation of parameter are introduced to solve for a solution of a non-homogeneous differential equation. Hence, the solution of equation (11) is

$$
\widetilde{E}_{g 2}(\lambda, \xi, \omega)=\chi_{1} \xi^{\phi}+\chi_{2} \xi^{-\phi}+\frac{i \omega \mu_{g}}{\alpha b^{2}} \int_{0}^{1} G(\xi ; \tau) \Psi(\tau) d \tau
$$

where $\Psi(\tau)=\int_{0}^{\infty} s \sigma_{0} e^{-\frac{(\ln \tau+b l)^{2}}{b}} E_{o b j}(s, \tau, \omega) J_{1}(\lambda s) d s$, and $G(\xi ; \tau)=\frac{\tau^{\phi} \xi^{-\phi}}{\tau}$ is the Green's function, $\phi=\lambda / b$ and $\chi_{1}$ and $\chi_{2}$ are arbitrary constants. We calculate the integration term of equation (13) by using method of subsection for which dividing the ground into $n$ disks and having the thickness equal to $d$ and assume that the disk is very thin. So, the current distribution on each disk may be approximated to be constant at each position of $z$. Consequently, equation (13) becomes

$$
\widetilde{E}_{g 2}(\lambda, \xi, \omega)=\chi_{1} \xi^{\phi}+\chi_{2} \xi^{-\phi}+\frac{i \omega \mu_{g}}{\alpha b} \sum_{j=1}^{n} G_{j}\left(\xi ; \xi_{j}\right) R\left(\lambda, \xi_{j}, \omega\right)\left(\xi_{j}\right)
$$

where $R\left(\lambda, \xi_{j}, \omega\right)=\int_{0}^{\infty} s p\left(s, \xi_{j}, \omega\right) J_{1}(\lambda s) d s$ and $p\left(s, \xi_{j}, \omega\right)=d \sigma_{0} e^{-\frac{\left(\ln \xi_{j}+b l\right)^{2}}{b}}$ $\times E_{j o b j}\left(s, \xi_{j}, \omega\right)$. By substituting (12) and (14) into (7), we derive the electric field as

$$
\widetilde{E}_{\text {ground }}(\lambda, \xi, \omega)=\kappa_{1} \xi^{\phi}+\kappa_{2} \xi^{-\phi}+\frac{i \omega \mu_{g}}{\alpha b} \sum_{j=1}^{n} G_{j}\left(\xi ; \xi_{j}\right) R\left(\lambda, \xi_{j}, \omega\right)\left(\xi_{j}\right),
$$

where $\kappa_{1}=\eta_{1}+\chi_{1}$ and $\kappa_{2}=\eta_{2}+\chi_{2}$ are arbitrary constants to be determined. Under the condition that $\widetilde{E}_{\text {ground }} \rightarrow 0$, then we require that $\kappa_{1}=0$. 
Consequently, equation (15) becomes

$$
\widetilde{E}_{\text {ground }}(\lambda, \xi, \omega)=\kappa_{2} \xi^{-\phi}+\frac{i \omega \mu_{g}}{\alpha b} \sum_{j=1}^{n} G_{j}\left(\xi ; \xi_{j}\right) R\left(\lambda, \xi_{j}, \omega\right)\left(\xi_{j}\right),
$$

The arbitrary constants $v$ from equation (6), $\kappa_{2}$ from equation (16) can be solved by imposing the continuity of $\widetilde{E}$ and $\frac{\partial \widetilde{E}}{\partial z}$ at the interface. At Air-Earth interface, $z=0$. Therefore, we have

$$
\widetilde{E}_{\text {air }}(\lambda, 0, \omega)=\widetilde{E}_{\text {ground }}(\lambda, 0, \omega) \text { and } \frac{\partial}{\partial z} \widetilde{E}_{\text {air }}(\lambda, 0, \omega)=\frac{\partial}{\partial z} \widetilde{E}_{\text {ground }}(\lambda, 0, \omega) .
$$

We obtain the electric field in air as

$$
\begin{aligned}
& E_{\text {air }}(r, z, \omega) \\
& =i \omega \mu_{g} a I(\omega) \int_{0}^{\infty}\left(e^{-\lambda|z+h|}+\frac{e^{-\lambda|h|+\lambda z}}{m_{g}+\lambda}\right) J_{1}(\lambda a) J_{1}(\lambda r) d \lambda \\
& \quad+i \omega \mu_{g} \int_{0}^{\infty} \sum_{j=1}^{n} s p\left(s, \xi_{j}, \omega\right) d s\left(\xi_{j}\right)^{\frac{\lambda}{b}} \int_{0}^{\infty}\left[\left(e^{\lambda l}-1\right) e^{\lambda z}\right] J_{1}(\lambda s) J_{1}(\lambda r) d \lambda .
\end{aligned}
$$

The electric field in ground can be written as

$$
\begin{aligned}
& E_{\text {ground }}(r, z, \omega) \\
& =i \omega \mu_{g} a I(\omega) \int_{0}^{\infty}\left(\frac{\xi^{\frac{\lambda}{b}}}{e^{\lambda h}}+\frac{e^{-\lambda l-\lambda|h|}}{\left(m_{g}+\lambda\right)} \xi^{-\frac{\lambda}{b}}\right) J_{1}(\lambda a) J_{1}(\lambda r) d \lambda \\
& \quad+i \omega \mu_{g} \int_{0}^{\infty} \sum_{j=1}^{n} s p\left(s, \xi_{j}, \omega\right) d s\left(\xi_{j}\right)^{\frac{\lambda}{b}} \int_{0}^{\infty}\left[e^{\lambda l}-\xi^{-\frac{\lambda}{b}}\right] J_{1}(\lambda s) J_{1}(\lambda r) d \lambda .
\end{aligned}
$$

By dividing each disk into $m$ rings and having the width equal to $\frac{\Theta}{m}=\Pi$ and $s_{i}$ is the radius of the $j^{t h}$ - ring from the center where $\Theta$ is a radius of the cylinder body. Rewriting equations (17) or (18) to find the electric field from each of the partition, and since

$$
\int_{0}^{\Theta} s S_{j} E_{j o b j}\left(s, \xi_{j}, \omega\right) J_{1}(\lambda s)=\sum_{i=1}^{m} s_{i} p\left(s, \xi_{j}, \omega\right) J_{1}\left(\lambda s_{i}\right)(\Pi),
$$

Then, we have

$$
E\left(r_{i^{\prime}}, \xi_{j^{\prime}}, \omega\right)
$$




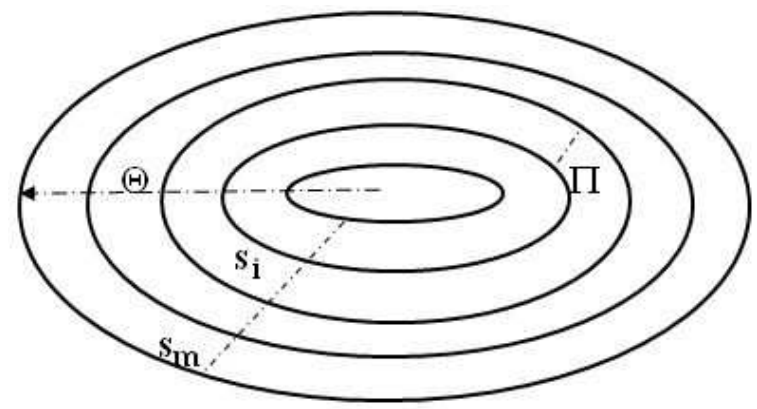

Figure 2: Geometric model for $i^{\text {th }}$ - ring of each disk.

$$
\begin{aligned}
= & \frac{i \omega \mu_{g} a^{2} I(\omega)}{2} \int_{0}^{\infty}\left(\xi_{j^{\prime}}{ }^{\frac{\lambda}{b}} e^{-\lambda|h|}+\frac{e^{-\lambda(2 l+|h|)}}{\left(m_{g}+\lambda\right)} \xi_{j^{\prime}}{ }^{-\frac{\lambda}{b}}\right) J_{1}\left(\lambda r_{i^{\prime}}\right) d \lambda \\
& +i \omega \mu_{g} \Pi \int_{0}^{\infty} \sum_{j=1}^{n} \sum_{i=1}^{m} s_{i} p\left(s_{i}, \xi_{j}, \omega\right)\left(\xi_{j}\right)^{\frac{\lambda}{b}}\left[e^{\lambda l}-\xi_{j^{\prime}}{ }^{-\frac{\lambda}{b}}\right] J_{1}\left(\lambda s_{i}\right) J_{1}\left(\lambda r_{i^{\prime}}\right) d \lambda,
\end{aligned}
$$

where $J_{1}(\lambda a) \approx \frac{\lambda a}{2}$ and $i^{\prime}, i=1,2, \ldots, m ; r_{i^{\prime}}=s_{i} ; j^{\prime}, j=1,2, \ldots, n$ and $\xi_{j}=$ $\xi_{j^{\prime}}$. By using $p\left(s, \xi_{j}, \omega\right)=d \sigma_{0} e^{-\frac{\left(\ln \xi_{j}+b l\right)^{2}}{b}} E_{j o b j}\left(s, \xi_{j}, \omega\right)$, equation (19) can be rewritten in the matrix form as

$$
\left[\Lambda_{i^{\prime} j^{\prime}}+\frac{\delta_{i^{\prime} j^{\prime}}}{S_{j^{\prime}}}\right]_{m \times m}\left[p\left(r_{i^{\prime}}, \xi_{j^{\prime}}, \omega\right)\right]_{m \times m}=\left[\Xi_{i^{\prime} j^{\prime}}\right]_{m \times m}
$$

where

$$
\begin{aligned}
\Lambda_{i^{\prime} j^{\prime}} & =i \int_{0}^{\infty}\left[\omega \mu_{g} \Pi \sum_{i=1}^{m} s_{i} \sum_{j=1}^{n}\left(\xi_{j}\right)^{\frac{\lambda}{b}}\left(e^{\lambda l}-\xi_{j^{\prime}}-\frac{\lambda}{b}\right) J_{1}\left(\lambda s_{i}\right)\right. \\
& \left.-\frac{a I(\omega)}{\sigma_{0}}\left(\omega \mu_{g} \sigma_{0} \xi_{j^{\prime}} \frac{\lambda}{b}+e^{-\lambda(2 l+|h|)}\left(\left(\left(\lambda^{4}+\left(\omega \mu_{g} \sigma_{0}\right)^{2}\right)^{(1 / 2)}-\lambda^{2}\right)^{(1 / 2)}\right)\right)\right] \\
& \times J_{1}\left(\lambda r_{i^{\prime}}\right) d \lambda \\
\Xi_{i^{\prime} j^{\prime}} & =\frac{a^{2} I(\omega)}{4 \sigma_{0}} \int_{0}^{\infty}\left(\left(\left(\lambda^{4}+\left(\omega \mu_{g} \sigma_{0}\right)^{2}\right)^{(1 / 2)}+\lambda^{2}\right)^{(1 / 2)}-2 \lambda\right) e^{-\lambda(2 l+|h|)} \\
& \times \xi_{j^{\prime}}-\frac{\lambda}{b} J_{1}\left(\lambda r_{i^{\prime}}\right) d \lambda
\end{aligned}
$$


and

$$
\delta_{i^{\prime} j^{\prime}}=\left\{\begin{array}{ll}
1 & , i^{\prime}=j^{\prime} \\
0 & , i^{\prime} \neq j^{\prime}
\end{array} .\right.
$$

Chave's algorithm [1] is applied to equations (21) and (22) to find a numerical solution of the inverse Hankel transforms of the electric field.

\section{Discussion and Conclusion}

In this paper, we show the mode to study the electric field responsed from the object buried under the ground. To formulate the problem, the Cauchy-Euler method, the method of variation of parameter and the integral equations are employed. We in the first place formulated the problem to obtain the electric field and could be used to solve for the electric fields on the ground surface. We next consider a model having a circular cylinder body buried under the ground. We limit the conductivity to be denoted by $\sigma(z)=\sigma_{0} e^{-b(z-l)^{2}}$, where $\sigma_{0}$ a is positive constant, $b$ is constant, and $l$ is positive which is used to locate the peak of the bulge. The solution of electric field obtained in this model is more complexed than the works done of Ketchanwit [3], Lee and Ignetik [4], Yooyuanyong and Chumchop [6], and Yooyuanyong and Siew [7] and. The graphs are shown the conducts of the electric field against source-receiver spacing $r$ at different depths. In the trials, we firstly set up the frequency $300 \mathrm{~Hz}$, the electric current of coil $I=1 \mathrm{amp}$, the radius of coil $a=1 \mathrm{~m}$, the thickness of disk $d=1 \mathrm{~m}$. By fixing the value of $\sigma_{0}=0.1 S m^{-1}, b=1 m^{-1}, l=2 m$, and the object located under the ground at $z=1,5 \mathrm{~m}$ while the values of $r$ are varied. That is, $r=10,20,30,40 m$ as shown in Figure $3 a$ and $3 b$. Furthermore, by increasing the values of $\sigma_{0}$ to $2 S \mathrm{~m}^{-1}$ and $5 \mathrm{Sm}^{-1}$, and $l=5 \mathrm{~m}$ as shown in Figures 4 5 , respectively. We also reduced the value of $b$ to $0.5 \mathrm{~m}^{-1}$ and $0.2 \mathrm{~m}^{-1}$. Graphs of modulus of electric field that we obtained are shown the oscillation related to Yooyuanyong and Siew [7] and tended to zero as shown in Figures $3-5$. A bulge conductivity profile and the deepness $z=z_{1}$ of the object under the ground resulted the oscillation of the graphs. The higher conductive ground profile will response a bigger curve of electric field as shown in Figure 3-5. As the value of $b$ is reduced, the field response is also decreased as we expected. 

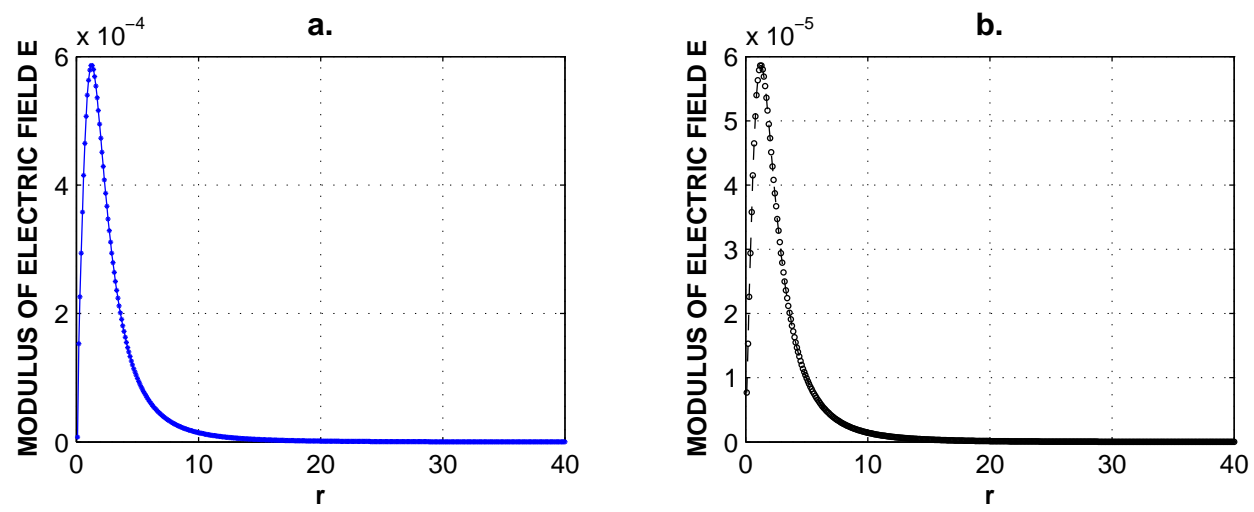

Figure 3: Graph of electric field $E$ versus $r$ for an earth structure model with a bulge conductivity ground profile which has an cylinder body buried under the ground with $\sigma_{0}=0.1 \mathrm{Sm}^{-1}, b=1 \mathrm{~m}^{-1}, l=2 \mathrm{~m}$, $r=10,20,30,40 m$, at $z=1,5 \mathrm{~m}$, the thickness of disk $d=1 \mathrm{~m}$, and frequency $=300 \mathrm{~Hz}$.
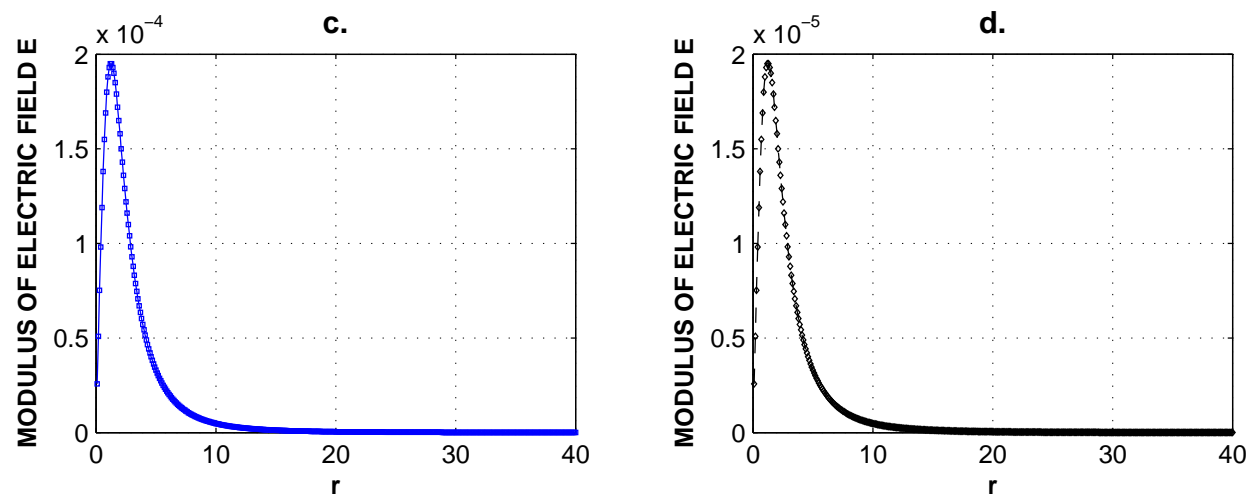

Figure 4: Graph of electric field $E$ versus $r$ for an earth structure model with a bulge conductivity ground profile which has an cylinder body buried under the ground with $\sigma_{0}=2 S \mathrm{~m}^{-1}, b=0.5 \mathrm{~m}^{-1}, l=2 \mathrm{~m}$, $r=10,20,30,40 m$, at $z=1,5 m$, the thickness of disk $d=1 \mathrm{~m}$, and frequency $=300 \mathrm{~Hz}$. 

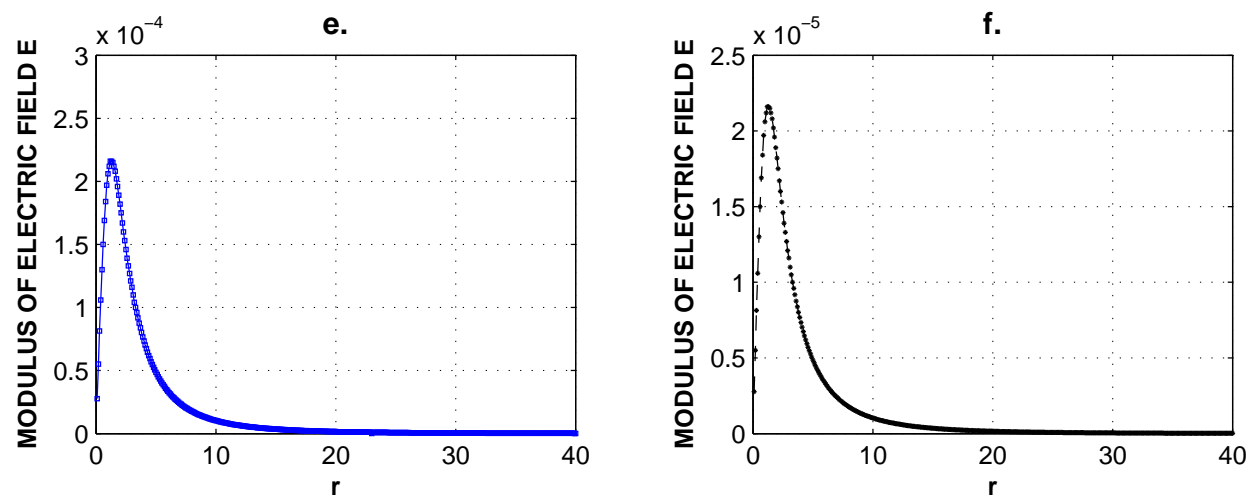

Figure 5: Graph of electric field $E$ versus $r$ for an earth structure model with a bulge conductivity ground profile which has an cylinder body buried under the ground with $\sigma_{0}=5 S m^{-1}, b=0.1 m^{-1}, l=5 m$, $r=10,20,30,40 \mathrm{~m}$, at $z=1,5 \mathrm{~m}$, the thickness of disk $d=1 \mathrm{~m}$, and frequency $=300 \mathrm{~Hz}$.

\section{Acknowledgments}

We would like to thank referees for their valuable comments and suggestions. This work is supported by Srinakharinwirot University, Thailand.(grant number $119 / 2557)$.

\section{References}

[1] A.D. Chave, " Numerical integration of related Hankel transforms by quadrature and continued fraction expansion", Geophysics 48(1983), 16711686.

[2] G. W. Hohmann and A. P. Raiche, Inversion of controlled-Source Electromagnetic Data, Electromagnetic methods in applied geophysics 1(1988), 469-503.(ed. Nabighian, M. N.)SEG

[3] P. Ketchanwit, Time domain electromagnetic response in heterogeneous media, M.Sc. Thesis, Silpakorn University, Thailand, 2001. 
[4] T.J. Lee and R. Ignetik, Transient Electromagnetic Response of a halfspace with Exponential Conductivity Profile and its Applications to Salinity Mapping, Exploration Geophysics 25(1994), 39-51.

[5] H.F. Morrison, R.J. Phillips and D.P. O'Brien, Quantitative interpretation of transient electromagnetic fields over a layered half-space, Geophysical prospecting, 21(1969), 1-20.

[6] S. Yooyuanyong and N. Chumchob, Mathematical modeling of electromagnetic sounding for a conductive 3-D circular cylinder body embedded in a conducting half-space, Proceedings of the third asian mathematical conference $2000(\mathbf{2 0 0 0})$, 590-603.

[7] S. Yooyuanyong and P. F. Siew, The electromagnetic response of a disk beneath an exponentially varying conductive overburden, J. Australian Mathematical Society Series B. 41(2000), E1-E28.

[8] S. Yooyuanyong, Electromagnetic response over a varying conductive ground, Songklanakarin, J. Sci. Technol. 22(4)(2000), 457-466. 
